\title{
Investigation of Mach cones and the corresponding two-particle correlations in a microscopic transport model
}

\author{
loannis Bouras* \\ Institut für Theoretische Physik, Johann Wolfgang Goethe-Universität, Max-von-Laue-Str. 1, \\ 60438 Frankfurt am Main, Germany \\ E-mail: bouras@th.physik.uni-frankfurt.de

\section{B. Betz} \\ Institut für Theoretische Physik, Johann Wolfgang Goethe-Universität, Max-von-Laue-Str. 1, \\ 60438 Frankfurt am Main, Germany \\ E-mail: betzath.physik.uni-frankfurt.de
}

\section{Z. Xu}

Institut für Theoretische Physik, Department of Physics, Tsinghua University, Beijing 100084, China

Collaborative Innovation Center of Quantum Matter, Beijing, China

E-mail: xuzhe@mail.tsinghua.edu.cn

\section{Greiner}

Institut für Theoretische Physik, Johann Wolfgang Goethe-Universität, Max-von-Laue-Str. 1, 60438 Frankfurt am Main, Germany

E-mail: carsten.greinereth.physik.uni-frankfurt.de

\begin{abstract}
We study the formation of Mach cones in a full $(3+1)$-dimensional setup of relativistic heavy-ion collisions, considering a transverse and longitudinal expanding medium at RHIC energies. For smooth initial conditions and central collisions we investigate the jet-medium interaction using high-energy jets and by varying the value of the shear viscosity over entropy density ratio, $\eta / s$. For small viscosities we observe the formation of Mach cones, while for larger viscosities the characteristic structures smear out and vanish eventually. We discuss the formation of a doublepeak structure both in a single and a multiple jet event.
\end{abstract}

52 International Winter Meeting on Nuclear Physics - Bormio 2014,

27-31 January 2014

Bormio, Italy

\footnotetext{
* Speaker.
} 


\section{Introduction}

Collisions of heavy nuclei both at the Relativistic Heavy-Ion Collider (RHIC) [1, 2] and the Large Hadron Collider (LHC) [3] indicate the formation of a new state of matter, the quark-gluon plasma (QGP). The large value of the measured elliptic flow coefficient, $v_{2}$, suggests that the QGP behaves like an almost perfect fluid as demonstrated by various calculations of viscous hydrodynamics [4] and microscopic transport models [5]. The values determined for the shear viscosity over entropy density ratio are approximately $\eta / s=0.1-0.2$ and thus close to the conjectured lower bound, $\eta / s=1 / 4 \pi$, derived via the correspondence between conformal field theory and string theory in an Anti-de-Sitter space [6].

High-energy jets traversing the expanding medium deposit energy and momentum. Due to this interaction with the bulk medium, those jets are (strongly) suppressed, a phenomenon that is called jet quenching [7]. Its explicit mechanisms of the jet-medium interaction are still a matter of research. The two- and three-particle correlations extracted from experiment show a complete suppression of the away-side jets for $3<p_{T}<6 \mathrm{GeV}$ and due to energy and momentum conservation the signal appears again in a lower- $p_{T}$ range, showing a double-peak structure [8]. The origin of the double-peak structure was discussed to be connected to Mach cones generated by fast partons traversing the strongly-coupled medium [9]. However, recent studies on the triangular flow and hot spots $[8,10]$ issue a further, probably more satisfactory explanation for the appearance of the double peak structure.

Up until today, however, the contribution of jet-induced Mach cones to the double-peak structure remains unclear. To gain a better understanding of the contribution of Mach cones to the double-peak structure in two-particle correlations, we investigate the evolution pattern of jet-induced Mach cones and their particle distrubtion in a relativistic, $(3+1)$-dimensional expanding system of a heavy-ion collision for various values of the shear viscosity over entropy density ration, $\eta / s$, using the microscopic transport model BAMPS (Boltzmann Approach of MultiParton Scatterings) [11].

In an earlier publication, [12], we already studied the formation of Mach cones using the above mentioned kinetic transport model applying a static box scenario and thus neglecting all effects from expansion. We showed that, considering a source term depositing both energy and momentum, the double-peak structure is overshadowed by the strong contribution of the head shock and diffusion wake, while the double-peak structure appears for a source term (that lacks physical motivation from any theoretical model) which leads to only energy but no momentum deposition. These results are consistent with an investigation based on an AdS/CFT calculation [13] and a hydrodynamic model $[14,15]$. The comparison with the latter publications proofs once more BAMPS can model a hydrodynamic expansion [16, 17]. Using the same non-expanding scenario, we studied the effect of dissipation in box scenario [12] and found that dissipation tends to destroy any Mach cone signal.

However, as discussed in Ref. [18], the flow-velocity profile created by jets in the transverse plane interacts with the radial flow of the background medium, changing e.g. the effective angle of a Mach cone. This effect of transverse expansion on Mach cones has also been studied in Refs. [19]. It was found that a double-peak structure can be created by averaging over different jet paths. The main contribution to the double-peak structure originates from jets which are deflected by radial 
flow. In the rare case that a jet traverses into the opposite direction of the radial flow, the interplay between radial flow and jets reduces the strong contribution of the diffusion wake and head shock and results in a double-peak structure for this single event.

In the following, we investigate a similar setup as in [19], but including not only transverse but also longitudinal expansion, exploring in particular the influence of viscosity. This work is based on the publication [20]. In this work, the units are $\hbar=c=k=1$.

\section{Numerical setup}

We use the framework of BAMPS in a fully $(3+1)$-dimensional setup designed for ultrarelativistic heavy-ion collisions [11]. Here, we treat a massless gluon gas as classical Boltzmann particles with a degeneracy factor of $g=16$. Particles only collide via binary collisions with an isotropic cross section, i.e., a cross section with an isotropic distribution of the collision angle. We perform the numerical calculations using a constant value of the shear viscosity over entropy density ratio, $\eta / s$. For isotropic binary collisions, the shear viscosity is given by $\eta=0.4 e /(n \sigma)$, where $e(n)$ is the local rest frame energy (particle) density.

As we intend to focus on the impact of transverse radial and longitudinal flow on jets, we neglect additional effects from density fluctuations. Likewise, we neglect any effects originating from elliptic flow and only consider central collisions. We apply smooth Glauber initial conditions in the transverse direction [21] and a Gaussian rapidity distribution in longitudinal direction as it was found at RHIC [22] that the rapidity distribution of charged hadrons can be described by a Gaussian. In beam $(z)$ direction, we determine the width of the nuclear overlap region based on the Lorentz-contracted nuclear thickness that is approximated by a Gaussian as well. For the high- $p_{T}$ region we apply a power law which approximately fits the $p+p$ data [23]. Thus, we apply the following parametrization for the initial non-thermal single-particle distribution function

$$
f(\vec{x}, \vec{p})=K \frac{1}{E}\left(\frac{Q^{n}}{Q^{n}+p_{T}^{n}}\right)^{m} \exp \left(-\frac{y_{\text {rap }}^{2}}{\sigma_{y}^{2}}\right) \exp \left(-\frac{z^{2}}{\sigma_{z}^{2}}\right) T_{\mathrm{A}}\left(x+\frac{b}{2}, y\right) T_{\mathrm{B}}\left(x-\frac{b}{2}, y\right),
$$

where $p_{T}=\sqrt{p_{x}^{2}+p_{y}^{2}}$ denotes the transverse momentum, $y_{\text {rap }}$ the momentum rapidity, and $b$ the impact parameter. The nuclear thickness function [24] $T_{\mathrm{A}}(x, y)=\int_{-\infty}^{+\infty} d z \rho_{\mathrm{A}}(x, y, z)$ is integrated in $z$-direction. $\rho_{\mathrm{A}}(\vec{x})$ denotes the Woods-Saxon distribution for the nucleus $A, \rho_{\mathrm{A}}(\vec{x})=$ $\rho_{0} /\left(1+\exp \left(\frac{|\vec{x}|-R_{\mathrm{A}}}{D}\right)\right)$. Here, $R_{\mathrm{A}}=1.12 A^{1 / 3}-0.86 A^{-1 / 3}$ and the mean density of the nucleus is $\rho_{0}=0.17 \mathrm{fm}^{-3}$. For the thickness parameter we use $D=0.54 \mathrm{fm}$. As we are studying RHIC energies, we exclusively use gold nuclei with a mass number of $A=B=197$ and choose $Q=1.3 \mathrm{GeV}$, $n=4, m=1.5, \sigma_{y}=1, \sigma_{z}=0.13 \mathrm{fm}$, and $K=0.0135$.

In the following we investigate scenarios where the jet is set on top of the bulk medium. Please note that the jet interacts with the same cross section as the medium particles. Similar to the numerical setup discussed in [19] we initialize the starting points of the jet on a semi-circle displayed in Fig. 1 with a radius of $r=4 \mathrm{fm}$ at mid-rapidity,

$$
\vec{x}_{\mathrm{jet}}=r\left(\begin{array}{c}
\cos \phi_{\mathrm{jet}} \\
\sin \phi_{\mathrm{jet}} \\
0
\end{array}\right) \text {. }
$$




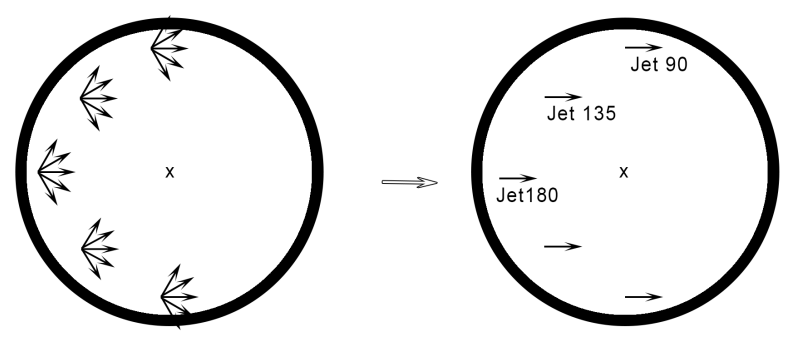

Figure 1: Schematic representation of different jet paths initialized on a semi-circle, see also Ref. [19]. The left panel shows all possible paths, the right panel the reduced number of paths due to reasons of symmetry.

While in the experiment back-to-back correlated jets are created due to momentum conservation, we assume that the near-side jet escapes rapidly to the vacuum and thus, we neglect the near-side jet contribution. In contrast, the away-side jet traverses the hot and dense medium of the collision. Due to reasons of symmetry, the possible jet paths that need to be studied in a central collision (see left panel of Fig. 1) reduces drastically (as shown in the right panel of Fig. 1). All jets studied here have an initial momentum only in the $x$-direction of $p_{x}=E_{\text {jet }}=20 \mathrm{GeV}$.

Although performing a full $(3+1)$-dimensional simulation, we restrict the following discussion on the midrapidity region. Thus, we extract all quantities and azimuthal particle distributions for a small space-time rapidity of $\left|\eta_{\text {rap }}\right|<0.1$. Since the parton cascade BAMPS has no effective hadronization process implemented yet, the final particle distribution is obtained by stopping the simulation at a certain time and extracting the macroscopic quantities as well as the azimuthal particle distribution from the final gluon-momentum distribution.

\section{Results}

In the following we study the evolution of jet-induced Mach cones in ultrarelativistic heavyion collisions. First we consider a jet starting at a fixed-angle position of $\phi_{\text {jet }}=180^{\circ}$ on a semicircle. In this particular case the jet initially propagates in opposite direction to the radial flow generated in a heavy-ion collision. We examine different values of the shear viscosity over entropy density ratio, reflecting different possible interaction strenghts with the medium. To illustrate the results we depict a time evolution of the local-rest frame energy-density profile around mid-rapidity, $\left|\eta_{\text {rap }}\right|<0.1$, in Fig. 2, overlaid with a velocity profile represented by scaled arrows.

At early times, $t \sim 1 \mathrm{fm} / c$, the energy density reaches very high values and a Mach cone has not yet built up. At later times, the energy density decreases drastically due to the longitudinal and the transverse expansion. A conical structure induced by the jet has developed at $t=5 \mathrm{fm} / c$ for $\eta / s=0.08$. In contrast, for larger values of $\eta / s$, such a structure has not built up (yet). The differences in the shape of the Mach cone for various $\eta / s$ becomes more evident at later times, $t=9 \mathrm{fm} / c$ and $t=12 \mathrm{fm} / c$. For the case with $\eta / s=0.08$ the initial jet has already lost almost all of its energy, i.e. has thermalized, within a very short time in the beginning of the simulation. As mentioned above we do not distinguish between bulk medium and jet particles, which implies that for $\eta / s=0.08$ the mean free path of the jet is very small. It is clear, that considering perturbatice 


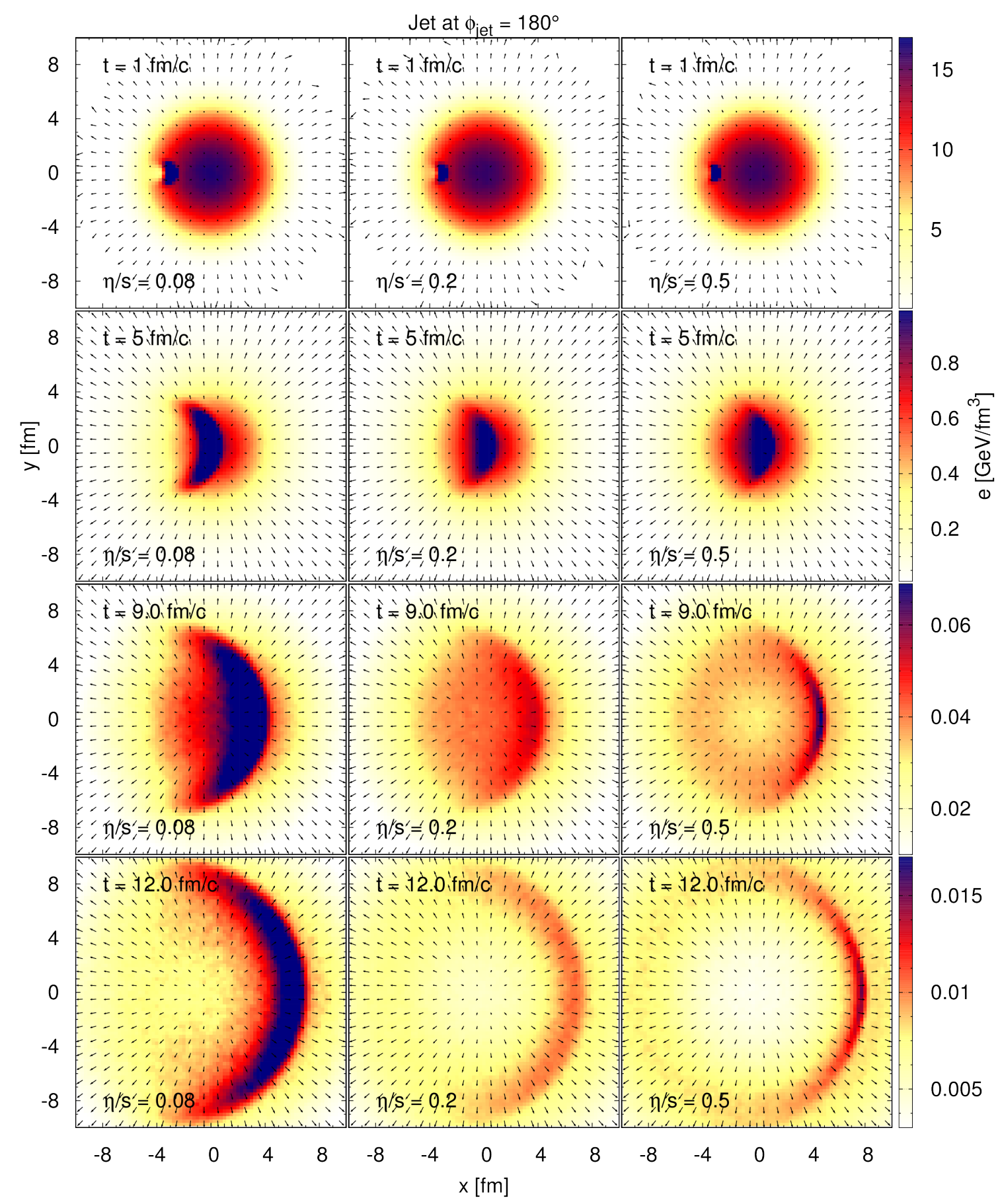

Figure 2: Time evolution of a Mach cone in a central heavy-ion collision. The local rest frame energy density around midrapidity $\left(\left|\eta_{\text {rap }}\right|<0.1\right)$ is shown, overlaid by the velocity profile, indicated by scaled arrows. The results are depicted at various time steps and different values of the shear viscosity over entropy density ratio, $\eta / s$. The jet is initialized at fixed-angle position of $\phi_{\text {jet }}=180^{\circ}$ on the semi-circle with an initial momentum of $p_{x}=E_{\mathrm{jet}}=20 \mathrm{GeV}$. 
QCD based elastic and inelastic processes [25] would change significantly the jet energy loss. However, such a treatment remains a future task.
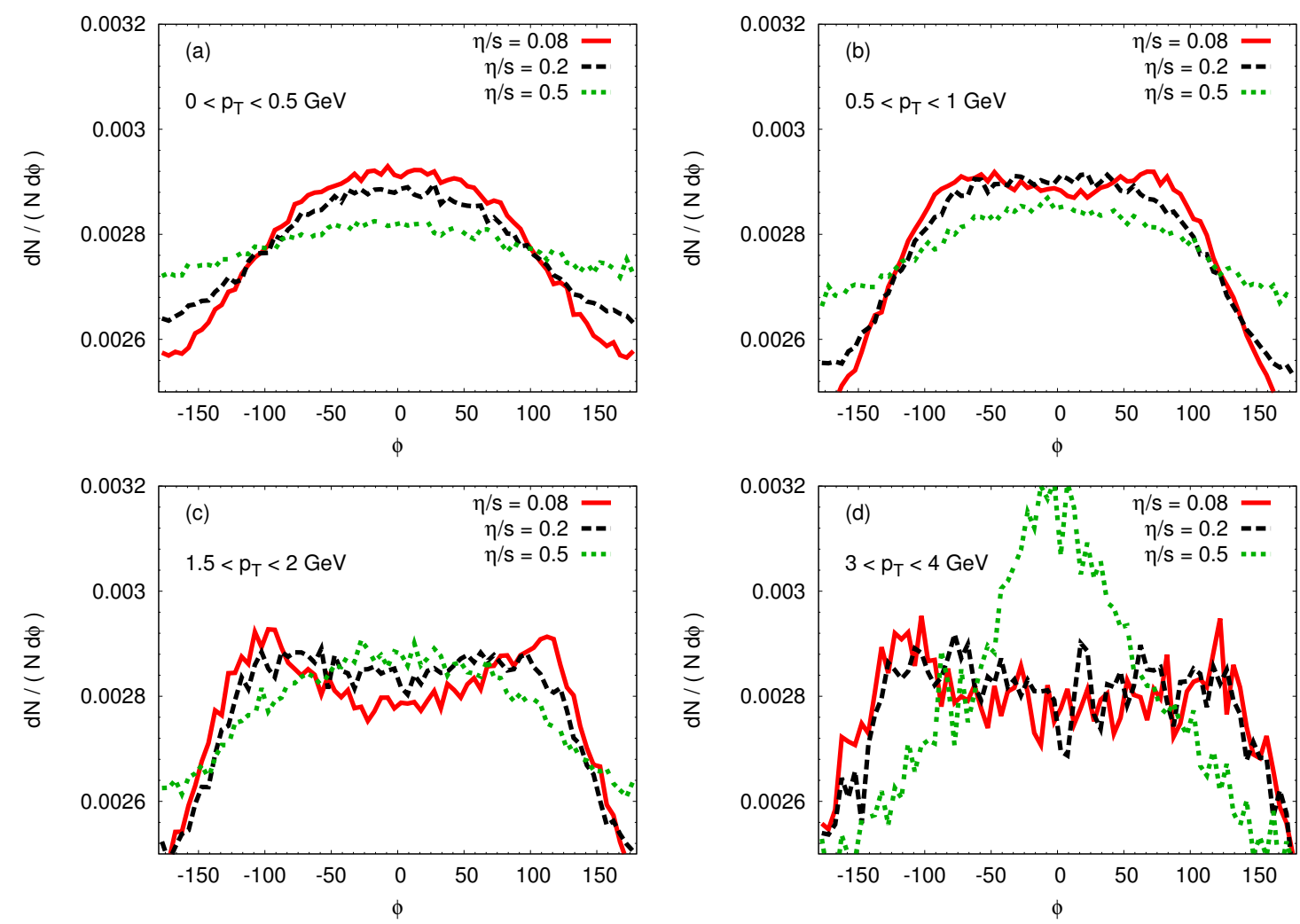

Figure 3: Two-particle correlations, $\mathrm{d} N /(N \mathrm{~d} \phi)$, extracted from BAMPS calculations for different values of $\eta / s$ and $p_{T}$-cuts. The jet is initialized at fixed-angle position of $\phi_{\text {jet }}=180^{\circ}$ on the semi-circle (see Fig. 2). The results are shown for a fixed time $t=12 \mathrm{fm} / c$.

The results in Fig. 2 indicate that a single jet propagating through the middle of the medium creates a Mach cone for moderate values of $\eta / s$ and sufficiently large times. The shock front, however, is strongly curved due to the interaction of the jet with the medium. The diffusion wake is not directly visible as it is superimposed by the radial flow of the background medium, indicating that the contribution of the diffusion wake and head shock is possibly reduced in the final particle distribution.

In Fig. 3, we show the extracted normalized azimuthal particle distribution $\mathrm{d} N /(N \mathrm{~d} \phi)$. The plot demonstrates that a double-peak structure can develop for a small value of the shear viscosity over entropy density ratio, $\eta / s$, and a $p_{T}$-cut of $p_{T}>0.5 \mathrm{GeV}$. For $\eta / s=0.08$ and $0.5<p_{T}<1$ $\mathrm{GeV}$ a double-peak structure is observed at $\phi \approx \pm 70^{\circ}$, suggesting that the contribution of head shock and diffusion wake is indeed compensated by the radial flow. For larger transverse momenta, however, [see e.g. Fig. 3, panel (d)] the double-peak structure appears around $\phi \approx \pm 120^{\circ}$. Here, this conical structure is due to the fact that matter flows into region of lower pressure and energy density behind the jet and is thus not directly a signal of the Mach cone. Finally, for $\eta / s=0.5$ the contribution from the head shock and diffusion wake is very strong, leading to a single peak in 
forward direction.

Considering a single jet with a fixed-angle position of $\phi_{\text {jet }}=135^{\circ}$ on the semi-circle, the shape of the Mach cone changes significantly. Due to the relative position on the semi-circle and the strong radial flow, the single jet is deflected, especially for small $\eta / s$. This deflection, however, reduces for large $\eta / s$ as the jet-medium interaction weakens, keeping the jet on its initial propagation direction.
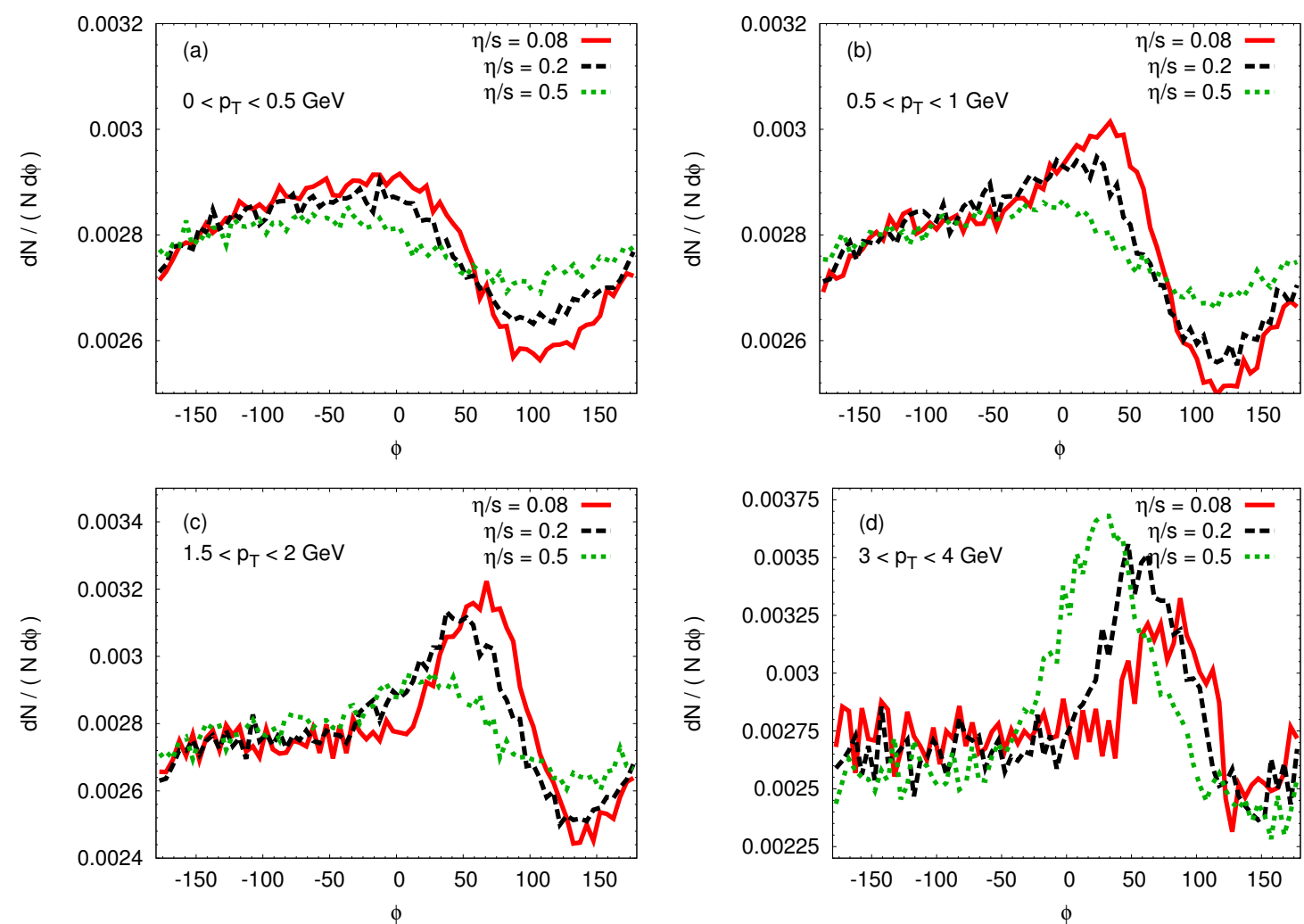

Figure 4: Two-particle correlations, $\mathrm{d} N /(N \mathrm{~d} \phi)$, extracted from BAMPS calculations for different values of $\eta / s$ and $p_{T}$-cuts. The jet is initialized at fixed-angle position of $\phi_{\mathrm{jet}}=135^{\circ}$ on the semi-circle. The results are shown for a fixed time $t=12 \mathrm{fm} / c$.

These results indicate that the generated diffusion wake and head shock are less compensated by the radial flow due to the fact the jet does not propagate in opposite direction to the radial flow. The deflection of the jet for $\eta / s=0.08$ leads to only one peak in the two-particle correlations as displayed in Fig. 4. This peak originates from the head shock and diffusion wake of the distorted Mach cone and becomes sharper and more pronounced for larger $p_{T}$. We also see that the position of the induced peaks in Fig. 4 changes with viscosity. The larger the value of $\eta / s$, the smaller the peak angle, reflecting the fact that the jet is less deflected for larger values of $\eta / s$.

Although the two-particle correlations determined for this specific single-jet scenario only exhibit one single peak, a double-peak structure is possible when considering in a second jet event in the lower semi-circle [19]. In this case, however, the double-peak is mainly generated due to the superposition of several deflected and distorted jet-induced Mach cones. I.e., the origin for the 

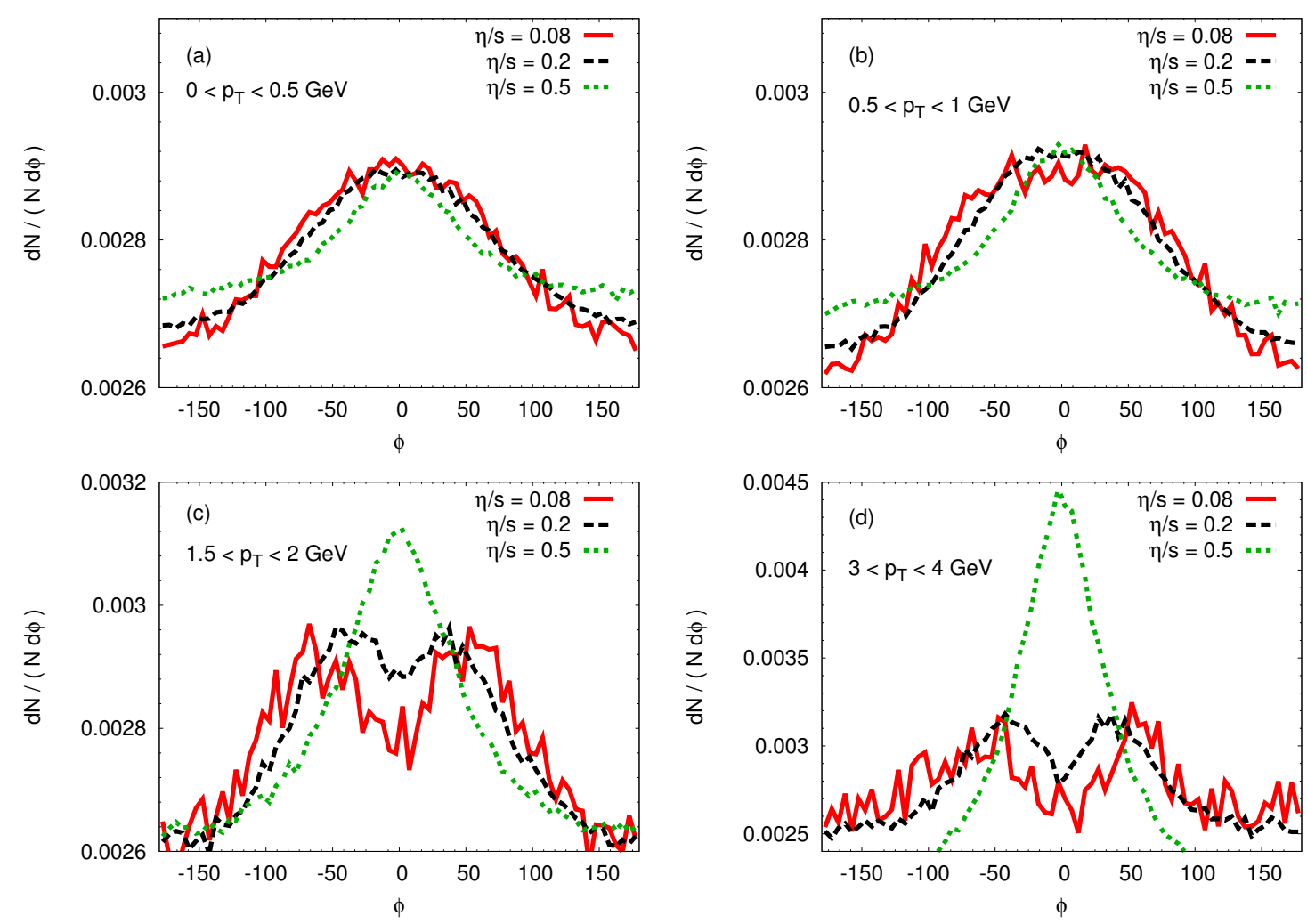

Figure 5: Two-particle correlations, $\mathrm{d} N /(N \mathrm{~d} \phi)$, extracted from BAMPS calculations for different values of $\eta / s$ and $p_{T}$-cuts. Here, we consider various starting positions of the jet on the semi-circle. The results are shown at a fixed time of $t=12 \mathrm{fm} / c$.

double-peak structure is not the Mach cone itself but the deflected head shocks and diffusion wakes of the distorted Mach cones as demonstrated below.

Averaging over all possible jet trajectories with randomly chosen starting positions on the semi-circle, i.e., $\phi_{\text {jet }}=90^{\circ}-270^{\circ}$, we get closest to the experimental situation. Here we consider many different jet events. Fig. 5 displays the normalized azimuthal particle distribution determined for this multiple-jet scenario. A double-peak structure appears for sufficiently high $p_{T}$ and low $\eta / s$ with peaks around $\phi \approx 50^{\circ}$. This result is in line with the Ref. [19] applying a hydrodynamic evolution.

\section{Conclusion}

The above results provide evidence that Mach cones can form in heavy-ion collision if the medium created has a small viscosity. However, as compared to an ideal, zero-viscosity medium, the pattern of the Mach cone is more curved. If a jet gets deflected by radial flow, the shape of the induced Mach cone is distorted. We demonstrate that the radial flow in a heavy-ion collision affects both, the final pattern of the Mach cone [18] and the observed particle distribution. Our results imply that the influence of the radial flow is most important for the observation of a double- 
peak structure. A large dissipation and/or large initial jet energy lead to a smaller influence of the radial flow. The effect of longitudinal flow is studied here in a qualitative way and was not considered previous studies [19]. We conclude that the longitudinal flow does not affect the results significantly.

In conclusion, we have shown that Mach cones can form in a longitudinal and transverse expanding heavy-ion collision. Considering multiple jet-events, a double-peak structure evolves if the energy over entropy-density ratio is $\eta / s<0.5$. Please note that the peak position does not change significantly with $\eta / s$. However, this double-peak structure does not originate from Mach cones but arises due to the contribution of the head shock and diffusion wake originating from the deflected jets $[18,19]$. Although initial-state fluctuations certainly provide a large contribution to the experimentally observed double-peak structure [10] it will be important to quantify the contribution from jets. The double-peak signal might not be an appropriate observable to disentangle both contributions [12] as it is mainly medium-driven. However, allocating a more appropriate signature remains an open challenge.

\section{Acknowledgements}

The authors are grateful to the Center for Scientific Computing (CSC) at Frankfurt University for providing the necessary computing resources. I. Bouras is acknowledges support from HGSHire. Z. Xu's work is supported partially by the Major State Basic Research Development Program in China (No. 2014CB845400) and by the National Natural Science Foundation of China projects (No. 11275103, 11335005). This work was supported by BMBF and the Helmholtz International Center for FAIR within the framework of the LOEWE program launched by the State of Hesse.

\section{References}

[1] John Adams et al. Experimental and theoretical challenges in the search for the quark gluon plasma: The STAR Collaboration's critical assessment of the evidence from RHIC collisions. Nucl.Phys., A757:102-183, 2005.

[2] K. Adcox et al. Formation of dense partonic matter in relativistic nucleus-nucleus collisions at RHIC: Experimental evaluation by the PHENIX collaboration. Nucl.Phys., A757:184-283, 2005.

[3] K Aamodt et al. Higher harmonic anisotropic flow measurements of charged particles in pb-pb collisions at $\sqrt{s_{N N}}=2.76$ TeV. Phys. Rev. Lett., 107:032301, Jul 2011.

[4] Matthew Luzum and Paul Romatschke. Conformal Relativistic Viscous Hydrodynamics: Applications to RHIC results at $\sqrt{s_{N N}}=200 \mathrm{GeV}$. Phys.Rev., C78:034915, 2008.

[5] Zhe Xu, Carsten Greiner, and Horst Stoecker. PQCD calculations of elliptic flow and shear viscosity at RHIC. Phys.Rev.Lett., 101:082302, 2008.

[6] P. Kovtun, D.T. Son, and A.O. Starinets. Viscosity in strongly interacting quantum field theories from black hole physics. Phys.Rev.Lett., 94:111601, 2005.

[7] J. Adams et al. Transverse momentum and collision energy dependence of high $\mathrm{p}(\mathrm{T})$ hadron suppression in $\mathrm{Au}+\mathrm{Au}$ collisions at ultrarelativistic energies. Phys.Rev.Lett., 91:172302, 2003.

[8] Fuqiang Wang. Novel Phenomena in Particle Correlations in Relativistic Heavy-Ion Collisions. Prog. Part. Nucl. Phys., 74:35-54, 2014. 
[9] Horst Stoecker. Collective flow signals the quark gluon plasma. Nucl.Phys., A750:121-147, 2005.

[10] Bjorn Schenke, Sangyong Jeon, and Charles Gale. Elliptic and triangular flow in event-by-event (3+1)D viscous hydrodynamics. Phys.Rev.Lett., 106:042301, 2011.

[11] Zhe Xu and Carsten Greiner. Thermalization of gluons in ultrarelativistic heavy ion collisions by including three-body interactions in a parton cascade. Phys.Rev., C71:064901, 2005.

[12] I. Bouras, A. El, O. Fochler, H. Niemi, Z. Xu, et al. Transition From Ideal To Viscous Mach Cones In A Kinetic Transport Approach. Phys.Lett., B710:641-646, 2012.

[13] Jorge Noronha, Miklos Gyulassy, and Giorgio Torrieri. Di-Jet Conical Correlations Associated with Heavey Quark Jets in anti-de Sitter Space/Conformal Field Theory Correspondence. Phys.Rev.Lett., 102:102301, 2009.

[14] Barbara Betz, Miklos Gyulassy, Jorge Noronha, and Giorgio Torrieri. Anomalous Conical Di-jet Correlations in pQCD vs AdS/CFT. Phys.Lett., B675:340-346, 2009.

[15] Barbara Betz, Jorge Noronha, Giorgio Torrieri, Miklos Gyulassy, Igor Mishustin, et al. Universality of the Diffusion Wake from Stopped and Punch-Through Jets in Heavy-Ion Collisions. Phys.Rev., C79:034902, 2009.

[16] I. Bouras, E. Molnar, H. Niemi, Z. Xu, A. El, et al. Investigation of shock waves in the relativistic Riemann problem: A Comparison of viscous fluid dynamics to kinetic theory. Phys.Rev., C82:024910, 2010.

[17] I. Bouras, E. Molnar, H. Niemi, Z. Xu, A. El, et al. Relativistic shock waves in viscous gluon matter. Phys.Rev.Lett., 103:032301, 2009.

[18] L.M. Satarov, H. Stoecker, and I.N. Mishustin. Mach shocks induced by partonic jets in expanding quark-gluon plasma. Phys.Lett., B627:64-70, 2005.

[19] Barbara Betz, Jorge Noronha, Giorgio Torrieri, Miklos Gyulassy, and Dirk H. Rischke. Universal Flow-Driven Conical Emission in Ultrarelativistic Heavy-Ion Collisions. Phys.Rev.Lett., 105:222301, 2010.

[20] I. Bouras, B. Betz, Z. Xu, and C. Greiner. Mach cones in viscous heavy-ion collisions. 2014.

[21] Michael L. Miller, Klaus Reygers, Stephen J. Sanders, and Peter Steinberg. Glauber modeling in high energy nuclear collisions. Ann.Rev.Nucl.Part.Sci., 57:205-243, 2007.

[22] I.G. Bearden et al. Charged meson rapidity distributions in central Au+Au collisions at $\mathrm{s}(\mathrm{NN}) * *(1 / 2)$ = 200-GeV. Phys.Rev.Lett., 94:162301, 2005.

[23] S.S. Adler et al. Mid-rapidity neutral pion production in proton proton collisions at $\sqrt{s}=200 \mathrm{GeV}$. Phys.Rev.Lett., 91:241803, 2003.

[24] P.F. Kolb, Ulrich W. Heinz, P. Huovinen, K.J. Eskola, and Kimmo Tuominen. Centrality dependence of multiplicity, transverse energy, and elliptic flow from hydrodynamics. Nucl.Phys., A696:197-215, 2001.

[25] Oliver Fochler, Jan Uphoff, Zhe Xu, and Carsten Greiner. Radiative parton processes in perturbative QCD - an improved version of the Gunion and Bertsch cross section from comparisons to the exact result. 2013. 